\title{
Operando characterization of battery anodes using mXRD and combined SAXS/WAXS
}

\section{Sandrine Lyonnard, Samuel Tardif}

\author{
CEA IRIG, Grenoble, France; \\ sandrine.lyonnard@cea.fr
}

Li-ion batteries are ubiquitous in our society. However, producing high performance, safe, and sustainable batteries remains a great challenge to foster the industrial development towards e-mobility, portable and stationnary applications. Materials engineering and new chemistries are key in this objective, as well as advanced characterization tools to probe the bulk \& interfacial properties of active materials. In particular, investigations in operando mode, e.g. during battery cycling under realistic conditions, are currently attracting an enormous interest. Synchrotron techniques have been widely employed to probe in real-time a large variety of battery technologies, e.g. Li-ion and beyond, to observe and map the evolving structures, in relation to materials composition \& design and battery operating conditions. In this talk, we will focus on the lithiation and ageing mechanisms in advanced electrodes, and show how operando X-rays (XRD/WAXS/SAXS) experiments can provide unique insights into the structural changes in graphite [1], silicon [2] and silicon-graphite [3-4] anodes with high time/spatial resolution. In particular, spatially-resolved mXRD gives access to 2D information in the depth of the electrode, as lithiation heterogeneities and phase distributions [1], while combined SAXS/WAXS allow to determine the sequential lithiation mechanism of active phases in a composite nanostructured material [3-4]. We will also adress the challenge to build beam-compatible battery cells, which is the pre-requisite to correlate real-time microscopic information to the electrochemical performance. Last, we will introduce the novel possibilities of performing 3D quantification of structural features evolutions in complex materials.

[1] S. Tardif et al, J. Mat. Chem. A, 2021.

[2] S. Tardif et al, ACS Nano, 2017, 11, 11306-11316.

[3] C. Berhaut et al, ACS Nano, 2019, 13, 10, 11538-11551.

[4] C. Berhaut et al, Energy Storage Materials, 2020

Keywords: Batteries, operando, synchrotron, neutron 